guidelines-was present in $29.4 \%$ and $19.8 \%$ of participants with and without SLE, respectively, and in $32.4 \%$ and $10.9 \%$ of participants with and without SLE, respectively, according to the modified $\mathrm{WHO}$ definition of the metabolic syndrome.

The second study included 8,807 participants sampled from the general US population, of whom $2.4 \%$ self-reported a doctor's diagnosis of gout. The prevalence of the metabolic syndrome, according to revised National Cholesterol Education Program Adult Treatment Panel III guidelines, was $62.8 \%$ and $25.4 \%$ among patients with and without gout, respectively.

These studies indicate that the metabolic syndrome might link increased cardiovascular risk and high levels of inflammation in patients with SLE, and that individuals with gout are likely to be at increased risk of cardiovascular disease and type 2 diabetes. A high prevalence of the metabolic syndrome in patients with these conditions has important therapeutic implications.

Original articles Chung CP et al. (2007) High prevalence of the metabolic syndrome in patients with systemic lupus erythematosus: association with disease characteristics and cardiovascular risk factors. Ann Rheum Dis 66: 208-214 Choi HK et al. (2007) Prevalence of the metabolic syndrome in patients with gout: the third National Health and

Nutrition Examination Survey. Arthritis Rheum 57: 109-115

\section{Cadherin-11 is a potential therapeutic target for the treatment of autoimmune arthritis}

Cadherins are integral transmembrane proteins that are involved in cell sorting, cell aggregation and tissue morphogenesis during embryogenesis, and postnatally, mediate cell-to-cell adhesion. These properties led Lee and colleagues to investigate cadherin molecule functioning in synovial lining and in autoimmune arthritis.

Initial investigations in healthy mice demonstrated that cadherin-11 is expressed in the synovium, and prompted further analysis of cadherin-11. A functional role of cadherin-11 in the synovium was indicated: in K/BxN transgenic mice (a model of autoimmune arthritis), cadherin-11 was strongly expressed on fibroblast-like synoviocytes in the synovial lining and the synovial pannus, was localized to adherens junctions, and was involved in homophilic adhesion by synovial fibroblasts. Analysis of synovium of cadherin-11-deficient mice, which demonstrated hypoplasia and had decreased synovial compaction, additionally confirmed this functional role.

The investigators then further explored the pathogenic implications of cadherin-11 in autoimmune arthrits. Using the K/BxN serum-transfer mode of autoimmune arthrits, it was shown that cadherin-11-deficient mice had a 50\% reduction in clinical arthritic activity. Assessment of cadherin-11 as a therapeutic target was also performed in mice with established arthritis, and administration of a monoclonal antibody towards cadherin-11 ameliorated the arthritis.

After further experiments, the investigators concluded that cadherin-11 is involved with the establishment and maintenance of normal synovium through cell-to-cell adhesion, compaction, and extracellular matrix production, and that cadherin-11 has a role in tissue remodelling, cartilage invasion, and influences fibroblast-like synoviocytes during an inflammatory reaction. This study demonstrates the possibility of using cadherin-11 as a therapeutic target for autoimmune arthritis.

Original article Lee DM et al. (2007) Cadherin-11 in synovial lining formation and pathology in arthritis. Science 315: $1006-1010$

\section{Environmental factors influence rheumatoid factor levels}

The presence of rheumatoid factor (RF) is used to diagnose and predict the prognosis of rheumatoid arthritis (RA), and is also associated with the polyarticular variant of juvenile idiopathic arthritis (JIA)-the subtype that most closely resembles adult-onset RA in terms of disease manifestation. The development of RA is affected by specific environmental factors. Young and colleagues evaluated perinatal and early childhood environmental risk factors for the presence of RF.

The authors measured RF levels in sera from 651 healthy children enrolled in the Diabetes Autoimmunity Study in the Young (DAISY) who were positive for HLA-DR4 genotypes and/or had a first-degree relative with type 1 diabetes. Data on infant diet and environmental factors were collected at ages 3, 6, 9, 12, and 15 months. A child was considered $\mathrm{RF}^{+}$if serum levels were $\geq 15 \mathrm{IU} / \mathrm{ml}$.

In total, 23 children in this cohort were $\mathrm{RF}^{+}$. Children who were negative for HLA-DR4 but $\mathrm{RF}^{+}$were less likely to have been breast-fed 